\title{
Riemann problem for constant flow with single-point heating source
}

\author{
Changsheng $\mathrm{Yu}^{1}$, Chengliang Feng ${ }^{1}$, Zhiqiang Zeng ${ }^{1}$, and Tiegang Liu ${ }^{1}$ \\ ${ }^{1}$ Beihang University
}

November 4, 2020

\begin{abstract}
This work focuses on the Riemann problem of Euler equations with global constant initial conditions and a single-point heating source, which comes from the physical problem of heating one-dimensional inviscid compressible constant flow. In order to deal with the source of Dirac delta-function, we propose an analytical frame of double classic Riemann problems(CRPs) coupling, which treats the fluids on both sides of the heating point as two separate Riemann problems and then couples them. Three structures of the exact Riemann solution are found, which is consistent with the results of numerical methods. Furthermore, we establish the uniqueness of the Riemann solution with some restrictions on the Mach number of the initial condition.
\end{abstract}

\section{Hosted file}

wileyNJD-AMA.pdf available at https://authorea.com/users/372928/articles/490754-riemannproblem-for-constant-flow-with-single-point-heating-source 